
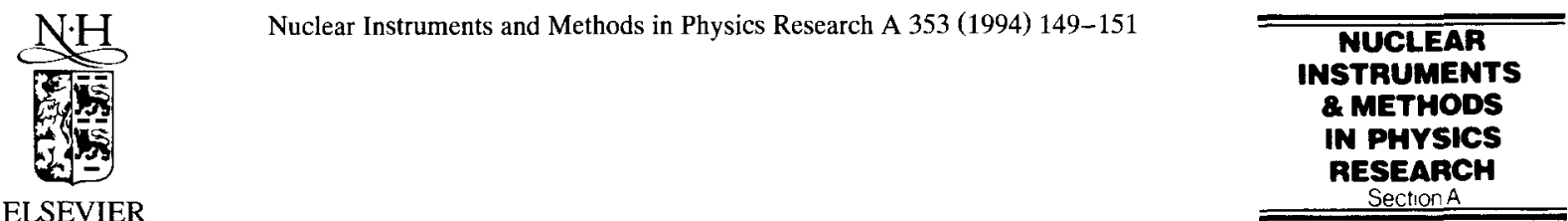

\title{
Water diffusion profile measurements in epoxy using neutron radiography
}

\author{
John T. Lindsay ${ }^{\mathrm{a}, *}$, Masahito Matsubayashi ${ }^{\mathrm{b}}$, Md. Nurul Islam ${ }^{\mathrm{c}}$ \\ ${ }^{a}$ Phoenix Memorial Laboratory, University of Michigan, Ann Arbor, MI, USA \\ b Japan Atomic Energy Research Institute, Tokal, Japan \\ ${ }^{c}$ Institute of Nuclear Science \& Technology, Atomic Energy Research Establishment, Bangladesh
}

\begin{abstract}
The diffusion characteristics of water in polymer materials have been studied for a few decades. Several methods have been developed to provide water diffusion characteristics as a function of time, temperature, pressure, or thickness of polymer. Unfortunately, most of these methods give the amount of water absorbed as a function of weight versus time at given environmental conditions. Concentration profiles of the water diffusion through the polymer have been unobtainable by these established methods. Neutron radiography is a method of non-destructive testing that has grown rapidly over the past ten years and is capable of giving these concentration profiles. Epoxy is one of the most commonly used polymers for which water diffusion information is important. In the automotive industry, epoxy is used both as a sealant and a bonder to prevent water from getting inside structures and causing corrosion. To prevent this corrosion, it is important to know the diffusion behavior of water in the epoxy adhesive.

This paper will demonstrate the use of high resolution neutron radiography as a viable method for the determination of the diffusion profile of water in commercially available epoxies. Aluminum coupons were constructed and joined together using four different epoxies. These coupons were then submerged in water. Neutron radiographs were made of the coupons as a function of total time submerged and water temperature. The weights of the coupons were also obtained as a function of submerged time for comparison with other methods. Four different epoxies were tested. Profiles of the water concentration are easily observed and measured.
\end{abstract}

\section{Introduction}

In earlier methods [1-6], the shape of the polymer sample was restricted to that required for each method such as film, membrane, sheet, or plate and it was impractical to apply the results to the actual case in which polymer materials are generally used with other materials and varied shapes.

Epoxy is one of the polymers used extensively as a bond and sealant in automobile doors. In this case epoxy adhesive also prevents water from getting into the door. Once water gets inside the door panel, it may cause the material to corrode. To prevent this corrosion, it is important to know the diffusion behavior of water in the epoxy adhesive. The epoxy is placed between several crimped layers of steel. This restricts the surface of the epoxy exposed to water and results in a narrow, long pathway for water diffusion to occur. To simulate this condition,

\footnotetext{
${ }^{*}$ Corresponding author.
}

coupons of aluminum were constructed and several different epoxies were used to bond these aluminum coupons together. These coupons were then placed in deionized water at various temperatures for increasing amounts of time. The samples were weighed to determine total water uptake and neutron radiography was used to measure the concentration profile of the water into the epoxy.

The purpose of this paper is to evaluate the permeation properties of water through various epoxy adhesives. In this study the permeation behavior of water was studied as a function of the immersion time, the water temperature, and the linear position of the water into the epoxy.

\section{Method}

Neutron radiography is analogous to X-ray radiography. Thermal neutrons interact with the nuclei of atoms whereas X-rays interact with the orbital shell electrons. In addition, hydrogen interacts quite well with neutrons having a large scatter plus absorption cross section for thermal neutrons. This makes neutrons ideally applicable for the measurement of water diffusion. 


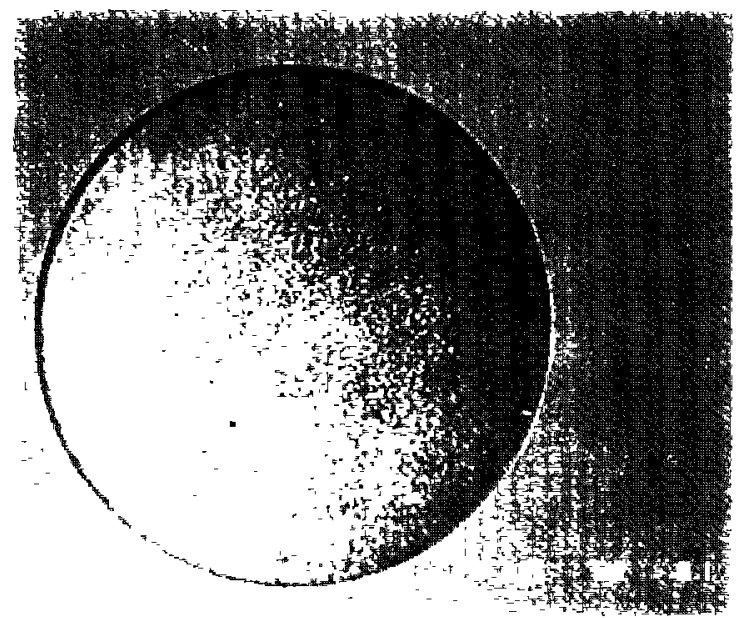

Fig. 1. Contrast enhanced, subtraction image for 200 hours immersion in $20^{\circ} \mathrm{C}$ water.

The approach used was to make high resolution neutron radiographs of the coupons prior to submersion in water to determine a base, or no water, image. The coupons were then immersed in water at various temperatures and then radiographed again. These radiographs were then placed on a light box and digitized using a Pixel Perceptics Pixel Pipeline processor installed in an Apple Quadra 950 using NIH's Image VDM software. By digitally subtracting these images, a resulting image of just the water could be obtained. Fig. 1 shows such an image. Fig. 1 is a contrast enhanced image of the difference between two digitized images (before and after) for a coupon after immersion for 200 hours in $20^{\circ} \mathrm{C}$ water. As can be seen the diffusion of water into the coupon can be quite easily seen. For comparisons with other methods the weight of the coupons were also determined for total water uptake as a function of time and temperature. A standard was also used during the radiography in order to determine the equivalent water thickness (water concentration) profile.
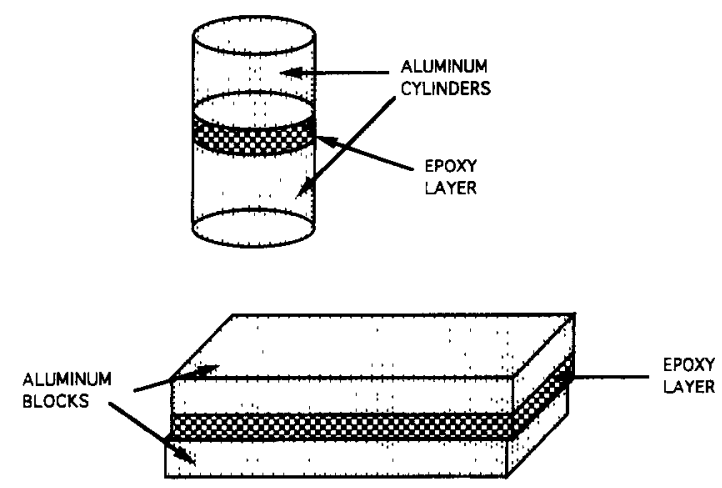

Fig. 2. Two different types of coupons used in this study.

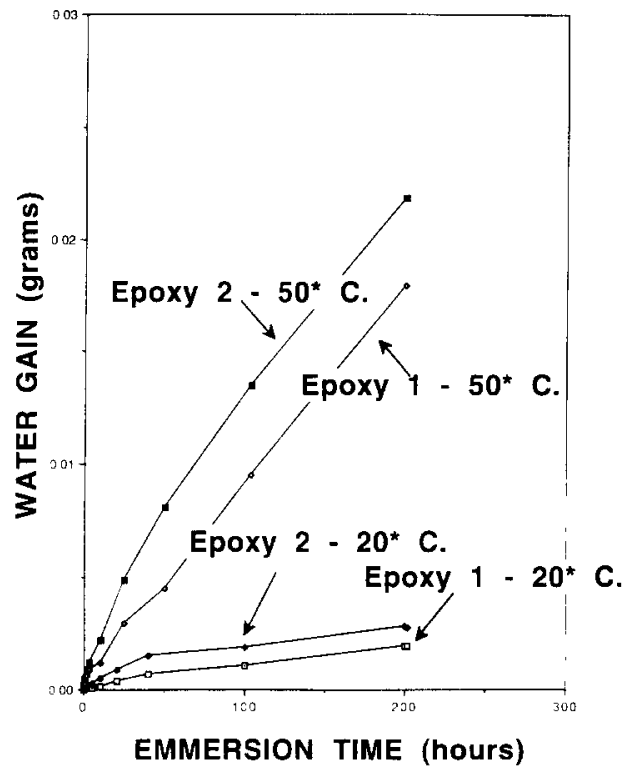

Fig. 3. Epoxy weight gain (grams of water) as a function of time and temperature.

Fig. 2 shows a drawing of the two different types of coupons used in this study. The original study was performed using circular coupons to match computer simulation programs already in use. This caused difficulty in matching the different radiographs exactly for subtraction purposes. The rectangular coupons were then constructed to make subtraction and mathematical modeling easier. The circular coupons were totally submerged in the water, whereas the rectangular coupons were only submerged one half of their length to facilitate removal of air during water diffusion.

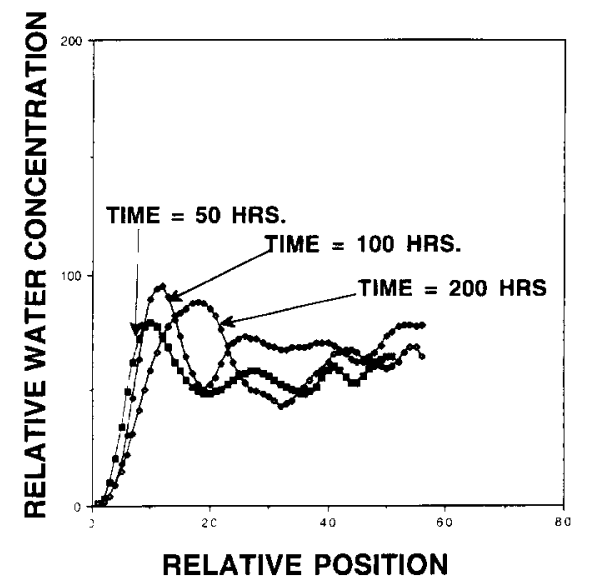

Fig. 4. Water concentration curves for epoxy 1 for different immersion times. 


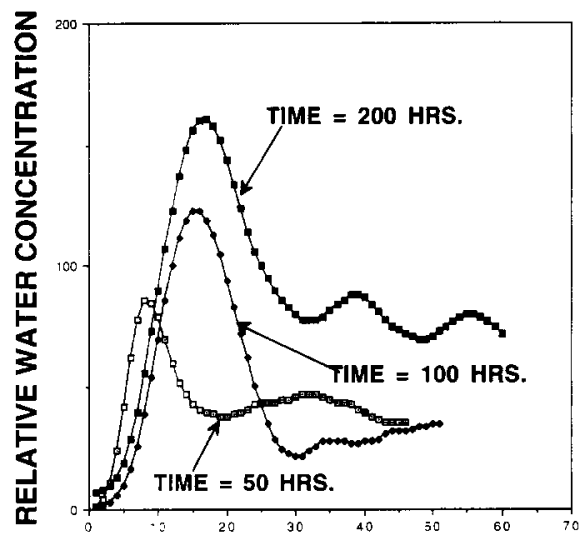

RELATIVE POSITION

Fig. 5. Water concentration curves for epoxy 1 for different immersion times.

The neutron radiographs were made using the high resolution vertical neutron radiography port installed in the Phoenix Memorial Laboratory, 2 MW Ford Nuclear Reactor Facility. This port gives a highly collimated $(L / D$ ratio $=340)$ and very pure (cadmium ratio $=200)$ thermal neutron beam (flux $=3.2 \times 10^{6} \mathrm{n} / \mathrm{cm}^{2} \mathrm{~s}$ ). Kodak Industrex SR film was used with a $25 \mu \mathrm{m}$ vapor deposited gadolinium metal converter screen for making the radiographs. The radiographs were then placed on a light box and digitized using a Perceptics Pixel Pipeline processor installed in an Apple Quadra 950 computer. NIH's Image VDM software was used to digitize, subtract, and enhance the radiographic images.

The coupons used in this study consisted of two aluminum structures bonded together with four different types of epoxy $(1,2,3,4)$. Multiple coupons were made using each epoxy. The coupons were then radiographed in order to determine if the epoxy bond was uniform (no or few voids) and complete. The coupons were then dried and cured at room temperature for 48 hours and then placed in a vacuum oven at $100^{\circ} \mathrm{C}$. The coupons were periodically removed and weighed until the curing was complete.

\section{Results}

Fig. 3 shows the weight gain of two different epoxies for two different temperatures. As can be seen the water uptake increased over time and was markedly different for different temperatures. These results agree with results published by earlier studies [1,2]. Figs. 4 and 5 show the relative water concentration as a function of position into the epoxy for two epoxies for different immerision times. The relative water concentration is shown to be due to problems with the water standard used. Future measurements will be conducted using a new standard.

\section{Conclusions}

As demonstrated, neutron radiography is easily capable of giving water concentration profiles and imaging water diffusion into epoxy layers. This work is continuing at PML to include water standards, develop diffusion models, and improve the technique.

\section{References}

[1] T.C. Wong and L.J. Broutman, Polymer Eng. Sci. 25(9) (1985).

[2] L.L. Marsh, R. Lasky, D.P. Sieraphim and G.S. Springer, IBM J. Res. Develop. 28 (November 1984).

[3] E. Sacher and J.R. Susko, J. Appl. Polymer Sci. 23 (1979) 2355.

[4] E. Sacher and J.R. Susko, J. Appl. Polymer Sci. 24 (1979) 1997.

[5] E. Sacher and J.R. Susko, J. Appl. Polymer Sci. 26 (1981) 679.

[6] Y.J. Chang, C.T. Chen and A.V. Tobolsky, J. Polymer Sci. 12 (1974) 1. 